J. Korean Math. Soc. 41 (2004), No. 6, pp. 1007-1021

\title{
ON ROTATION SURFACES IN THE MINKOWSKI 3-DIMENSIONAL SPACE WITH POINTWISE 1-TYPE GAUSS MAP
}

\author{
Athoumane Niang
}

\begin{abstract}
In this paper, we study rotation surfaces in the Minkowski 3-dimensional space with pointwise 1-type Gauss map and obtain by the use of the concept of pointwise finite type Gauss map, a characterization theorem concerning rotation surfaces and constancy of the mean curvature of certain open subsets on these surfaces.
\end{abstract}

\section{Introduction}

Recently in (2000), and in the framework of the theory of finite type submanifolds (see [2], [3]), the authors of [8] raising the following problem: classify all submanifolds in an $m$-Euclidean space $\mathbb{E}^{m}$ (or in the Minkowski space $\mathbb{E}_{1}^{m}$ ) satisfying the following equation

$$
\Delta G=f G,
$$

where $\Delta$ in the Laplacian of the induced metric and $G$ the Gauss map for the submanifold, for some function $f$ on the submanifold. The authors of [8] have studied ruled surfaces in 3-dimensional Minkowski space $\mathbb{E}_{1}^{3}$ with pointwise 1-type Gauss map, and obtain a classification theorem for them. Also, submanifolds in pseudo-Euclidean space with finite type Gauss map are studied (cf. [1], [5] among others). In the paper [6] a characterization of the helicoid as ruled surfaces with pointwise 1-type Gauss map in 3-dimensional Euclidean space is obtained. On the other hand, Chen and Piccini [4] made a general study on submanifolds of Euclidean space with finite type Gauss map and classified the compact surfaces of 1-type Gauss map.

Received May 29, 2003.

2000 Mathematics Subject Classification: 53B25, 53C40.

Key words and phrases: Gauss map, Laplacian, pointwise 1-type. 
In the paper [9] we studied rotation surfaces in 3-dimensional Euclidean space and obtained the following result.

THEOREM 1.1. Let $M$ be a connected surface of revolution in a 3 -dimensional Euclidean space $\mathbb{E}^{3}$ whose axis of rotation is $\mathcal{L}$. Let $M^{\prime}$ be any connected component of the subset $M-\mathcal{L}$. Then we have: $M^{\prime}$ is pointwise $1-$ type Gauss map if and only if $M^{\prime}$ a constant mean curvature.

In this paper we use the concept of pointwise 1-type Gauss map introduced in [8] to obtain the lorentz version of the above theorem:

THEOREM 1.2. Let $M$ be a connected semi-riemmannian surface of revolution in a 3 -dimensional Minkowski space $\mathbb{E}_{1}^{3}$ whose axis of rotation is $\mathcal{L}$. Let $M^{\prime}$ be any connected component of the subset $M-\mathcal{L}$. Then we have: $M^{\prime}$ is pointwise 1- type Gauss map if and only if $M^{\prime}$ a constant mean curvature.

Throughout this paper, we assume that all surfaces $M$ are connected, and all objects are at least of class $C^{3}$. Here we will agree to interchange the notation of vectors by rows and there notions by columns

\section{Preliminaries}

The 3-dimensional Minkowski space $\mathbb{E}_{1}^{3}$ is given with its canonical metric $d x^{2}+d y^{2}-d z^{2}$ which we will denote by: $\langle$,$\rangle . For two tangent$ vectors $V=\left(X_{1}, X_{2}, X_{3}\right)$ and $W=\left(Y_{1}, Y_{2}, Y_{3}\right)$ of its tangent space, we have:

$$
\langle V, W\rangle=X_{1} Y_{1}+X_{2} Y_{2}-X_{3} Y_{3} .
$$

The cross product $V \times W$ of the vectors $V$ and $W$ is given by

$$
V \times W=\left(X_{2} Y_{3}-X_{3} Y_{2},-X_{1} Y_{3}+X_{3} Y_{1},-X_{1} Y_{2}+X_{2} Y_{1}\right) \text {. }
$$

By surface in the 3-dimensional Minkowski space $\mathbb{E}_{1}^{3}$ we mean an isometric immersion into $\mathbb{E}_{1}^{3}$ of a connected 2-dimensional pseudo-riemannian manifold $M$.

Let $M$ be a surface in the 3 -dimensional Minkowski space $\mathbb{E}_{1}^{3}$. The Gauss map $G$ sends each point of $M$ to a unit normal vector to $M$. We have, $G: M \rightarrow S$ where

$$
S=\left\{\begin{array}{c}
S_{1}^{2}(1) \\
\text { or } \\
H^{2}(1) .
\end{array}\right.
$$


The surface $M$ is lorentzian when $G$ takes its values in $S_{1}^{2}(1)$ and riemannian when $G$ takes its values in $H^{2}(-1)$.

Now we are going to recall some fundamental formulas to be used latter in this work. Assume that the surface $M$ in $\mathbb{E}_{1}^{3}$ is described locally by an isometric immersion

$$
\begin{array}{ccc}
X: \mathcal{U} \subset \mathbb{R}^{2} & \rightarrow & \mathbb{E}_{1}^{3} \\
(s, v) & \rightarrow & X(s, v),
\end{array}
$$

where $(s, v)$ are local coordinates on the open set $\mathcal{U}$ of $\mathbb{R}^{2}$.

Then on $\mathcal{U}$ the Gauss map $G$ is given by the followings formula:

$$
G=\frac{X_{s} \times X_{v}}{\left\|X_{s} \times X_{v}\right\|} \text {. }
$$

The first and the second fundamental forms $I$ and $I I$, respectively, are obtained on $\mathcal{U}$ by the following formulas:

$$
\left\{\begin{array}{l}
I=\left\langle X_{s}, X_{s}\right\rangle d s^{2}+2\left\langle X_{s}, X_{v}\right\rangle d s d v+\left\langle X_{v}, X_{v}\right\rangle d v^{2} \\
I I=\left\langle G, X_{s s}\right\rangle d s^{2}+2\left\langle G, X_{s v}\right\rangle d s d v+\left\langle G, X_{v v}\right\rangle d v^{2} .
\end{array}\right.
$$

The mean curvature $H$ is given on $\mathcal{U}$ by the following formula:

$$
2 H=\frac{\left\langle G, X_{s s}\right\rangle \cdot\left\langle X_{v}, X_{v}\right\rangle-2\left\langle G, X_{s v}\right\rangle \cdot\left\langle X_{s}, X_{v}\right\rangle+\left\langle G, X_{s s}\right\rangle \cdot\left\langle X_{s}, X_{s}\right\rangle}{\left\langle X_{s}, X_{s}\right\rangle \cdot\left\langle X_{v}, X_{v}\right\rangle-\left(\left\langle X_{s}, X_{v}\right\rangle\right)^{2}} .
$$

We end this preliminaries by recalling the formula for the Laplacian. In local coordinates $\left(x_{1}, x_{2}\right)$ on a the domain $\mathcal{U}$ for a surface $\mathrm{M}$ furnished with pseudo-riemannian metric say $g$ given by its matrix $\left(g_{i j}\right)$ with inverse matrix $\left(g^{i j}\right)$, the Laplacian $\Delta$ of $M$ is

$$
\Delta=-\frac{1}{\sqrt{\left|\operatorname{det}\left(g_{i j}\right)\right|}} \Sigma \frac{\partial}{\partial x^{i}}\left(\sqrt{\left|\operatorname{det}\left(g_{i j}\right)\right|} g^{i j} \frac{\partial}{\partial x^{j}}\right) .
$$

\section{Rotation surfaces in $\mathbb{E}_{1}^{3}$}

We will adopt the method of describing rotation surface in $\mathbb{E}_{1}^{3}$ given in [7]. There are three types of 1-parameter subgroups of isometries of the Minkowski 3-dimensional space $\mathbb{E}_{1}^{3}$ that leave a line pointwise fixe. 
There are called hyperbolic, elliptic or parabolic rotations depending whether the line fixed(axis of rotation) is spacelike, timelike or lightlike.

(i) A space line is congruent to the line: $y=z=0$. The corresponding subgroup of rotations around this axis consists of

$$
\left(\begin{array}{rrr}
1 & 0 & 0 \\
0 & \cosh (\varphi) & \sinh (\varphi) \\
0 & \sinh (\varphi) & l \cosh (\varphi)
\end{array}\right)
$$

$-\infty<\varphi<\infty$.

(ii) A timelike line is congruent to the $z$-axis. The subgroup of rotations around this axis consists of

$$
\left(\begin{array}{ccc}
\cos (\theta) & -\sin (\theta) & 0 \\
\sin (\theta) & \cos (\theta) & 0 \\
0 & 0 & 1
\end{array}\right),
$$

$0 \leq \theta \leq 2 \pi$

(iii) A lightlike line is congruent to the line: $x=0, y=z$. The subgroup of rotations around this axis consists of

$$
\left(\begin{array}{lll}
1 & -t & t \\
t & 1-\frac{t^{2}}{2} & \frac{t^{2}}{2} \\
t & -\frac{t^{2}}{2} & 1+\frac{t^{2}}{2}
\end{array}\right),
$$

$-\infty<t<\infty$.

A surface is called a surface of revolution if its image is stable under a 1-parameter subgroup of isometries which leave a line pointwise fixed. This general definition will be related to the ordinary one in terms of rotating a profile curve which lies in a certain plane containing the axis of rotation (see [7]).

For a surface of revolution corresponding to an axis $\mathcal{L}$, Let $M^{\prime}$ be any connected component of the subset $M-\mathcal{L}$. We have the following lemma from [7].

Lemma 3.1. (i) If $\mathcal{L}$ is spacelike, then $M^{\prime}$ is expressed in the form $x=g(s), y=r(s) \sinh (\varphi), z=r(s) \cosh (\varphi) ; a<s<b,-\infty<\varphi<\infty$ whith metric

$$
I=\left(g^{\prime 2}-r^{\prime 2}\right) d s^{2}+r^{2} d \varphi^{2}
$$

where $g$ and $r$ are smooth functions of the parameter $s$ such that

$$
g^{\prime 2}(s)-r^{2}(s) \neq 0 \text { and } r(s) \neq 0 \text {, and for all } s .
$$


(ii) If $\mathcal{L}$ is timelike, then $M^{\prime}$ is expressed in the form

$$
x=r(s) \cos (\theta), y=r(s) \sin (\theta), z=g(s) ; a<s<b, 0 \leq \theta \leq 2 \pi
$$

whith metric

$$
I=\left(r^{\prime 2}-g^{\prime 2}\right) d s^{2}+r^{2} d \theta^{2}
$$

where $g$ and $r$ are smooth functions of the parameter $s$ such that

$$
g^{\prime 2}(s)-r^{\prime 2}(s) \neq 0 \text { and } r(s) \neq 0 \text {, and for all } s .
$$

(iii) If $\mathcal{L}$ is timelike, then $M^{\prime}$ is expressed in the form

$$
\begin{aligned}
& x=t(g-f) \\
& y=f+\frac{t^{2}}{2}(g-f) \\
& z=g+\frac{t^{2}}{2}(g-f) ; \\
& a<s<b,-\infty<t<\infty
\end{aligned}
$$

whith metric

$$
I=\left(f^{\prime 2}-g^{\prime 2}\right) d s^{2}+\left(f^{2}-g^{2}\right) d t^{2},
$$

where $f$ and $g$ are smooth functions of the parameter such that

$$
g^{\prime 2}(s)-f^{\prime 2}(s) \neq 0 \text { and } g(s)-f(s) \neq 0, \text { and for all } s .
$$

Conversely, a surface given in the above form in each case is a surface of revolution ; the profile curve is $s \rightarrow x=g(s), z=r(s)$ in case (i) ; $s \rightarrow x=r(s), z=g(s)$ in case (ii) ; $s \rightarrow y=f(s), z=g(s)$ in case (iii) (see [7]).

In addition to above lemma we have the followings results.

LEMMA 3.2. (a) For a surface of revolution given in (i) in the lemma 3.1 and expressed in the form

$$
X(s, \varphi)=(z(s), x(s) \sinh (\varphi), x(s) \cosh (\varphi)),
$$

we have, when the profile curve is parametrised by the arc length $s$ and when we assume $x=x(s)>0$, the following results.

-(a1) The first and the second fundamental forms are given by

$$
\left\{\begin{array}{l}
I=\varepsilon_{1} d s^{2}+x^{2} d \varphi^{2} \\
I I=\varepsilon_{1}\left(z^{\prime} x^{\prime \prime}-x^{\prime} z^{\prime \prime}\right)+\left(z^{\prime} x\right) d \varphi^{2}
\end{array}\right.
$$

where $\varepsilon_{1}= \pm 1$. 
-(a2) The mean curvature $H$ satisfy

$$
\left\{\begin{array}{l}
2 H=\varepsilon_{1}\left(z^{\prime} x^{\prime \prime}-x^{\prime} z^{\prime \prime}\right)+\left(\frac{z^{\prime}}{x}\right) \\
2 H^{\prime}=\varepsilon_{1}\left(z^{\prime} x^{\prime \prime \prime}-x^{\prime} z^{\prime \prime \prime}\right)+\left(\frac{z^{\prime}}{x}\right)^{\prime}
\end{array}\right.
$$

-(a3) The Laplacian is given by

$$
\Delta=-\left(\varepsilon_{1}\left(\frac{\partial^{2}}{\partial s^{2}}+\frac{x^{\prime}}{x} \frac{\partial}{\partial s}\right)+\frac{1}{x^{2}} \frac{\partial^{2}}{\partial \varphi^{2}}\right) .
$$

(b) For a surface of revolution given in (ii) in the lemma 3.1 and expressed in the form

$$
X(s, \theta)=(x(s) \cos (\theta), x(s) \sin (\theta), z(s)),
$$

we have, when the profile curve is parametrised by the arc length $s$ and when we assume $x=x(s)>0$, the following results.

-(b1) The first and the second fundamental forms are given by

$$
\left\{\begin{array}{l}
I=\varepsilon_{1} d s^{2}+x^{2} d \theta^{2} \\
I I=\varepsilon_{1}\left(z^{\prime \prime} x^{\prime}-z^{\prime} x^{\prime \prime}\right)+\left(z^{\prime} x\right) d \theta^{2}
\end{array}\right.
$$

where $\varepsilon_{1}= \pm 1$.

-(b2) The mean curvature $H$ satisfy

$$
\left\{\begin{array}{l}
2 H=\varepsilon_{1}\left(z^{\prime \prime} x^{\prime}-z^{\prime} x^{\prime \prime}\right)+\left(\frac{z^{\prime}}{x}\right) \\
2 H^{\prime}=\varepsilon_{1}\left(z^{\prime \prime \prime} x^{\prime}-z^{\prime} x^{\prime \prime \prime}\right)+\left(\frac{z^{\prime}}{x}\right)^{\prime} .
\end{array}\right.
$$

-(b3) The Laplacian is given by

$$
\Delta=-\left(\varepsilon_{1}\left(\frac{\partial^{2}}{\partial s^{2}}+\frac{x^{\prime}}{x} \frac{\partial}{\partial s}\right)+\frac{1}{x^{2}} \frac{\partial^{2}}{\partial \theta^{2}}\right) .
$$

(c) And for a surface of revolution given in (iii) in the lemma 3.1 and expressed in the form

$$
X(s, t)=\left(t(z(s)-y(s)) ; y(s)+\frac{t^{2}}{2}(z(s)-y(s)) ; z(s)+\frac{t^{2}}{2}(z(s)-y(s)),\right.
$$

we have, when the profile curve is parametrised by the arc length $s$ and when we assume $z(s)-y(s)>0$, the following results.

-(c1) The first and the second fundamental forms are given by

$$
\left\{\begin{array}{l}
I=\varepsilon_{1} d s^{2}+(z-y)^{2} d t^{2} \\
I I=\varepsilon_{1}\left(z^{\prime} y^{\prime \prime}-y^{\prime} z^{\prime \prime}\right)+(z-y)\left(z^{\prime}-y^{\prime}\right) d t^{2}
\end{array}\right.
$$

where $\varepsilon_{1}= \pm 1$. 
-(c2) The mean curvature $H$ satisfy

$$
\left\{\begin{array}{l}
2 H=\varepsilon_{1}\left(z^{\prime} y^{\prime \prime}-y^{\prime} z^{\prime \prime}\right)+\left(\frac{z^{\prime}-y^{\prime}}{z-y}\right) \\
2 H^{\prime}=\varepsilon_{1}\left(z^{\prime} y^{\prime \prime \prime}-y^{\prime} z^{\prime \prime \prime}\right)+\left(\frac{z^{\prime}-y^{\prime}}{z-y}\right)^{\prime} .
\end{array}\right.
$$

-(c3) The Laplacian is given by

$$
\Delta=-\left(\varepsilon_{1}\left(\frac{\partial^{2}}{\partial s^{2}}+\frac{z^{\prime}-y^{\prime}}{z-y} \frac{\partial}{\partial s}\right)+\frac{1}{(z-y)^{2}} \frac{\partial^{2}}{\partial \varphi^{2}}\right) .
$$

Proof. We begin by one remark. The surfaces of revolution given above in 3.1, 3.5, and 3.9 are particular cases of the surfaces of revolution given in the lemma 3.1 in (i), (ii), and (iii), respectively. Now we have to proof the relations given in (a),(b), and (c). Consider first the case of surfaces de revolution in (a):

$$
X(s, \varphi)=(z(s), x(s) \sinh (\varphi), x(s) \cosh (\varphi)) .
$$

In (a1) we only have to proof the second formula for the seconde fundamental $I I$, since the first formula of first form fundamental $I$ is a particular case of (i) when the profile curve is now parametrised by its arc length $s$. By the following relations

$$
\begin{gathered}
X_{s}=\left(z^{\prime}, x^{\prime} \sinh (\varphi), x^{\prime} \cosh (\varphi),\right. \\
X_{\varphi}=(0, x \cosh (\varphi), x \sinh (\varphi)
\end{gathered}
$$

we get

$$
X_{s} \times X_{\varphi}=\left(-x x^{\prime}, x z^{\prime} \sinh (\varphi), x z^{\prime} \cosh (\varphi)\right),
$$

where we get the Gauss G

$$
G=-\left(x^{\prime}, z^{\prime} \sinh (\varphi), z^{\prime} \cosh (\varphi)\right) \text { and }\langle G, G\rangle=-\varepsilon_{1} .
$$

With the Gauss map $G$ so determined, we use again the relations above to find the followings vectors:

$$
\begin{gathered}
X_{s s}=\left(z^{\prime \prime}, x^{\prime \prime} \sinh (\varphi), x^{\prime \prime} \cosh (\varphi)\right), \\
X_{s \varphi}=\left(0, x^{\prime} \cosh (\varphi), x^{\prime} \sinh (\varphi)\right) \\
X_{\varphi \varphi}=\left(0, x^{\prime} \sinh (\varphi), x^{\prime} \cosh (\varphi)\right) .
\end{gathered}
$$

Then we find the functions

$$
e:=\left\langle G, X_{s s}\right\rangle=z^{\prime} x^{\prime \prime}-x^{\prime} z^{\prime \prime} ; g:=\left\langle G, X_{\varphi \varphi}\right\rangle=x z^{\prime} ; \quad f:=\left\langle G, X_{s \varphi}\right\rangle=0 \text {. }
$$

From these quantities we get easily the formula for II by using the formula given in 2.4. And using 2.5 we get the formula for the mean curvature $H$ and see easily the formula for its derivative $H^{\prime}$. To find the Laplcian $\Delta$ we apply the formula 2.5 with the metric $I$. Therefore we have proved relations in (a1). 
As we have seen, the prove the remain relations in (b) and (c), we search in each case, the Gauss map $G$, and the corresponding three vectors as above, that is, the vectors $X_{s s}, X_{s \theta}$, and $X_{\theta \theta}$ for (b), and the vectors $X_{s s}, X_{s t}$, and $X_{t t}$ for (c). An easy computation give the following relations for surfaces of revolution of type(b) and type(c). By using respectively 3.5 and 3.9 we have.

- For type (b): From

$$
X_{s}=\left(\begin{array}{c}
x^{\prime} \cos (\theta) \\
x^{\prime} \sin (\theta) \\
z^{\prime}
\end{array}\right), X_{\theta}=\left(\begin{array}{c}
-x \sin (\theta) \\
x \cos (\theta) \\
0 .
\end{array}\right)
$$

we get the following

$$
\begin{gathered}
G=-\left(\begin{array}{c}
z^{\prime} \cos (\theta) \\
z^{\prime} \sin (\theta) \\
x^{\prime}
\end{array}\right) ; X_{s s}=\left(\begin{array}{c}
x^{\prime \prime} \cos (\theta) \\
x^{\prime \prime} \sin (\theta) \\
z^{\prime \prime}
\end{array}\right) ; \\
X_{s \theta}=\left(\begin{array}{c}
-x^{\prime} \sin (\theta) \\
x^{\prime} \cos (\theta) \\
0
\end{array}\right) ; X_{\theta \theta}=\left(\begin{array}{c}
-x^{\prime} \cos (\theta) \\
-x^{\prime} \sin (\theta) \\
0
\end{array}\right) .
\end{gathered}
$$

- For type (c): From

$$
X_{s}=\left(\begin{array}{c}
t\left(z^{\prime}-y^{\prime}\right) \\
y^{\prime}+\frac{t^{2}}{2}\left(z^{\prime}-y^{\prime}\right) \\
z^{\prime}+\frac{t^{2}}{2}\left(z^{\prime}-y^{\prime}\right)
\end{array}\right), X_{t}=\left(\begin{array}{c}
(z-y) \\
t(z-y) \\
t(z-y) .
\end{array}\right)
$$

we get the following

$$
\begin{gathered}
G=-\left(\begin{array}{c}
-t\left(z^{\prime}-y^{\prime}\right) \\
z^{\prime}-\frac{t^{2}}{2}\left(z^{\prime}-y^{\prime}\right) \\
y^{\prime}+\frac{t^{2}}{2}\left(z^{\prime}-y^{\prime}\right)
\end{array}\right) ; X_{s s}=\left(\begin{array}{c}
t\left(z^{\prime \prime}-y^{\prime \prime}\right) \\
y^{\prime \prime}+\frac{t^{2}}{2}\left(z^{\prime \prime}-y^{\prime \prime}\right) \\
z^{\prime \prime}+\frac{t^{2}}{2}\left(z^{\prime \prime}-y^{\prime \prime}\right)
\end{array}\right) \\
X_{s t}=\left(\begin{array}{c}
0 \\
(z-y) \\
(z-y) ;
\end{array}\right) X_{t t}=\left(\begin{array}{c}
0 \\
(z-y) \\
(z-y)
\end{array}\right)
\end{gathered}
$$

From these vectors one gets easily the results in (b) and (c).

\section{The proof of the theorem}

In order to proof the theorem, we proceed in the following fashion. We have to prove a first part, that is, for a given connected component $M^{\prime}$ of a surface revolution $M$ of type (i) or (ii) or (iii) in lemma 3.1, 
the mean curvature $H$ is a constant when $M^{\prime}$ is pointwise 1-type Gauss map. And in a second part, we have to proof the converse for $M^{\prime}$ (in each of three types). Now we consider some facts and remarks.

- We have to consider, for a surface $M$ of type (i) any connected component $M^{\prime}$ of $\{M-\mathcal{L}$, with $x=r(s)>0\}$ or of $\{M-\mathcal{L}$, with $x=$ $r(s)<0\}$.

And for a surface $M$ of type (ii) or (iii) we have to consider any connected component $M^{\prime}$ of $\{M-\mathcal{L}$ with $z=r(s)>0\}$ or of $\{M-$ $\mathcal{L}$, with $x=r(s)<0\}$ and respectively, of $\{M-\mathcal{L}$, with $z-y=$ $g(s)-f(s)>0\}$ or of $\{M-\mathcal{L}$, with $z-y=g(s)-f(s)<0\}$. And in each of these six cases we have prove that the mean curvature is constant when the component $M^{\prime}$ is pointwise 1-type Gauss map.

Now let us make some remarks.

One can see that is not necessary in proving the constancy of $H$ to consider in each type the two components given above in each type, we are going to consider only one the them, and this will be the way we follow here. In particular this justified the surfaces considered in lemma 3.2. A last remark is: we will agree to prove here the complete result for types (i) and (iii); for (ii) it is not difficult to see that things work the same. After these remarks we can begun proofs.

Step1: case (i) We consider a surface of revolution $\mathrm{M}^{\prime}$ in those of type (a) in lemma 3.2, that is as in 3.1. Then $M^{\prime}$ is a connected component of the set $\{M-\mathcal{L}$, with $x(s)>0\}$. Let's express the condition $\Delta G=f G$ on $M^{\prime}$ for the Gauss map $G=-\left(x^{\prime}, z^{\prime} \sinh (\varphi), z^{\prime} \cosh (\varphi)\right)$.

We get from $G$ the following three vectors :

$$
\begin{aligned}
G_{s} & =-\left(x^{\prime \prime}, z^{\prime \prime} \sinh (\varphi), z^{\prime \prime} \cosh (\varphi)\right) . \\
G_{s s} & =-\left(x^{\prime \prime \prime}, z^{\prime \prime \prime} \sinh (\varphi), z^{\prime \prime \prime} \cosh (\varphi)\right) . \\
G_{\varphi \varphi} & =-\left(0, z^{\prime} \sinh (\varphi), z^{\prime} \cosh (\varphi)\right) .
\end{aligned}
$$

Then, the Laplacian of the Gauss map by applying the formula 3.4 is the vector:

$$
-\left[\varepsilon_{1}\left(G_{s s}+\frac{x^{\prime}}{x} G_{s}\right)+\frac{1}{x^{2}} G_{\varphi \varphi}\right] .
$$


So we get easily that

$$
\Delta G=\left\{\begin{array}{l}
\varepsilon_{1}\left(x^{\prime \prime \prime}+\frac{x^{\prime}}{x} x^{\prime \prime}\right) \\
\left\{\varepsilon_{1}\left(z^{\prime \prime \prime}+\frac{x^{\prime}}{x} z^{\prime \prime}\right)+\frac{1}{x^{2}} z^{\prime}\right\} \sinh (\varphi) \\
\left\{\varepsilon_{1}\left(z^{\prime \prime \prime}+\frac{x^{\prime}}{x} z^{\prime \prime}\right)+\frac{1}{x^{2}} z^{\prime}\right\} \cosh (\varphi) .
\end{array}\right\}
$$

From this formula it is convenient to introduce the following functions. Let $A:=x^{\prime \prime \prime}+\frac{x^{\prime}}{x} x^{\prime \prime}$ and $B:=\varepsilon_{1}\left(z^{\prime \prime \prime}+\frac{x^{\prime}}{x} z^{\prime \prime}\right)+\frac{1}{x^{2}} z^{\prime}$. Then we can write that

$$
\Delta G=\left(\varepsilon_{1} A, B \sinh (\varphi), B \cosh (\varphi)\right)
$$

From which we get the quantity:

$$
\langle\Delta G, G\rangle=-\left(\varepsilon_{1} x^{\prime} A-z^{\prime} B\right) .
$$

In fact, the condition $\Delta G=f G$ is equivalent to the condition

$$
\Delta G+\varepsilon_{1}\langle\Delta G, G\rangle G=0 .
$$

This condition is then equivalent to the following three equations:

$$
\left\{\begin{array}{l}
\varepsilon_{1} A-\varepsilon_{1}\left(\varepsilon_{1} x^{\prime} A-z^{\prime} B\right)\left(-x^{\prime}\right)=0 \\
\left\{B-\varepsilon_{1}\left(\varepsilon_{1} x^{\prime} A-z^{\prime} B\right)\left(-z^{\prime}\right)\right\} \sinh (\varphi) \\
\left\{B-\varepsilon_{1}\left(\varepsilon_{1} x^{\prime} A-z^{\prime} B\right)\left(-z^{\prime}\right)\right\} \sinh (\varphi) .
\end{array}\right.
$$

These are equivalent to the two followings equations:

$$
\text { (*) }\left\{\begin{array}{l}
\left(1+\varepsilon_{1} x^{2}\right) A-x^{\prime} z^{\prime} B=0 \\
x^{\prime} z^{\prime} A+\left(1-\varepsilon_{1} z^{\prime 2}\right) B=0 .
\end{array}\right.
$$

Better, by using the relation

$$
(r): z^{\prime 2}-x^{2}=\varepsilon_{1}
$$

we see that the condition in $(\star)$ is then equivalent to the following

$$
(\star \star)\left\{\begin{array}{l}
z^{\prime}\left(z^{\prime} A-\varepsilon_{1} x^{\prime} B\right)=0 \\
x^{\prime}\left(z^{\prime} A-\varepsilon_{1} x^{\prime} B\right)=0 .
\end{array}\right.
$$

By using once again the relation ( $r$ ) and its derivative, and after an easy computation, we get the following:

$$
z^{\prime} A-\varepsilon_{1} x^{\prime} B=\left(z^{\prime} x^{\prime \prime \prime}-x^{\prime} z^{\prime \prime \prime}\right)+\varepsilon_{1}\left(\frac{z^{\prime}}{x}\right)^{\prime} .
$$

-(We have omitted this computation in order to avoid the proof to be so long, but in step 2 below for surfaces of type (iii) we give the complete computation). 
On the other hand, from the second formula in 3.3 for derivative of mean curvature $H$ we get:

$$
\left(z^{\prime} x^{\prime \prime \prime}-x^{\prime} z^{\prime \prime \prime}\right)=2 \varepsilon_{1} H^{\prime}-\varepsilon_{1}\left(\frac{z^{\prime}}{x}\right)^{\prime}
$$

And inserting the left member of this relation in 4.3 , we see that:

$$
z^{\prime} A-\varepsilon_{1} x^{\prime} B=2 \varepsilon_{1} H^{\prime}
$$

Now the condition 4.2 becomes:

$$
\left\{\begin{array}{c}
z^{\prime} H^{\prime}=0 \\
x^{\prime} H^{\prime}=0
\end{array}\right.
$$

From this we get with the relation (r) that $H^{\prime 2}$ is zero, therefore $H^{\prime}$ is identically zero on $M^{\prime}$.

Conversely, assume that surface $M^{\prime}$ of type (a) in lemma 3.2 has constant mean curvature. We have to show how we can obtain the condition 4.2. In fact we have the relation:

$$
\left(z^{\prime} x^{\prime \prime \prime}-x^{\prime} z^{\prime \prime \prime}\right)=2 \varepsilon_{1} H^{\prime}-\varepsilon_{1}\left(\frac{z^{\prime}}{x}\right)^{\prime}
$$

And the relation

$$
z^{\prime} A-\varepsilon_{1} x^{\prime} B=\left(z^{\prime} x^{\prime \prime \prime}-x^{\prime} z^{\prime \prime \prime}\right)+\varepsilon_{1}\left(\frac{z^{\prime}}{x}\right)^{\prime} .
$$

Since $H^{\prime}=0$, then we get from these two relations that:

$$
z^{\prime} A-\varepsilon_{1} x^{\prime} B=0
$$

To get the two equations of the condition 4.2 , we multiply the equation above by $z^{\prime}$ and by $x^{\prime}$, respectively. This proves the theorem in case of (i).

Step2: case (iii) We consider a surface $M$ in the type (iii), therefore as in case(c) of lemma 3.2. And we assume as in step1, that the set $M^{\prime}$ is pointwise 1-type Gauss map. As before, we consider $M^{\prime}$ a connected component of the set $\{M-\mathcal{L}$, with $z(s)-y(s)>0\}$.

Since

$$
G=-\left(-t\left(z^{\prime}-y^{\prime}\right) ; z^{\prime}-\frac{t^{2}}{2}\left(z^{\prime}-y^{\prime}\right) ; y^{\prime}+\frac{t^{2}}{2}\left(z^{\prime}-y^{\prime}\right)\right)
$$


by setting $U:=z^{\prime}-y^{\prime}$, one get easily the following vectors:

$$
\begin{gathered}
G=-\left(\begin{array}{c}
-t U^{\prime} \\
z^{\prime}-\frac{t^{2}}{2} U^{\prime} \\
y^{\prime}-\frac{t^{2}}{2} U^{\prime}
\end{array}\right) ; G_{s}=\left(\begin{array}{c}
-t U^{\prime \prime} \\
z^{\prime \prime}-\frac{t^{2}}{2} U^{\prime \prime} \\
y^{\prime \prime}-\frac{t^{2}}{2} U^{\prime \prime}
\end{array}\right) ; \\
G_{s s}=\left(\begin{array}{c}
-t U^{\prime \prime \prime} \\
z^{\prime \prime}-\frac{t^{2}}{2} U^{\prime \prime \prime} \\
y^{\prime \prime}-\frac{t^{2}}{2} U^{\prime \prime \prime}
\end{array}\right) ; G_{t t}=\left(\begin{array}{c}
0 \\
-U \\
-U
\end{array}\right) .
\end{gathered}
$$

Then, the Laplacian of the Gauss map by applying the formula 3.12 is the vector:

$$
-\left[\varepsilon_{1}\left(G_{s s}+\frac{U^{\prime}}{U} G_{s}\right)+\frac{1}{U^{2}} G_{\varphi \varphi}\right] .
$$

So we get easily that

$$
\begin{aligned}
\Delta G & =\left(\begin{array}{l}
\varepsilon_{1} t\left(U^{\prime \prime \prime}+\left(\frac{U^{\prime}}{U}\right) U^{\prime \prime}\right) \\
\varepsilon_{1}\left(-z^{\prime \prime \prime}+\frac{t^{2}}{2} U^{\prime \prime \prime}\right)+\varepsilon_{1}\left(-z^{\prime \prime}+\frac{t^{2}}{2} U^{\prime \prime}\right)\left(\frac{U^{\prime}}{U}\right)+\frac{U^{\prime}}{U^{2}} \\
\varepsilon_{1}\left(-y^{\prime \prime \prime}+\frac{t^{2}}{2} U^{\prime \prime \prime}\right)+\varepsilon_{1}\left(-y^{\prime \prime}+\frac{t^{2}}{2} U^{\prime \prime}\right)\left(\frac{U^{\prime}}{U}\right)+\frac{U^{\prime}}{U^{2}}
\end{array}\right) \\
= & \left(\begin{array}{l}
\varepsilon_{1} t\left(U^{\prime \prime \prime}+\left(\frac{U^{\prime}}{U}\right) U^{\prime \prime}\right) \\
\left\{\varepsilon_{1}\left(-z^{\prime \prime \prime}-z^{\prime \prime}\left(\frac{U^{\prime}}{U}\right)\right)+\frac{U^{\prime}}{U^{2}}\right\}+\frac{t^{2}}{2} \varepsilon_{1}\left(U^{\prime \prime \prime}+\left(\frac{U^{\prime}}{U}\right) U^{\prime \prime}\right) \\
\varepsilon_{1} t\left(U^{\prime \prime \prime}+\left(\frac{U^{\prime}}{U}\right) U^{\prime \prime}\right) \\
\left\{\varepsilon_{1}\left(-y^{\prime \prime \prime}-y^{\prime \prime}\left(\frac{U^{\prime}}{U}\right)\right)+\frac{U^{\prime}}{U^{2}}\right\}+\frac{t^{2}}{2} \varepsilon_{1}\left(U^{\prime \prime \prime}+\left(\frac{U^{\prime}}{U}\right) U^{\prime \prime}\right)
\end{array}\right) .
\end{aligned}
$$

In the view of this formula it is convenient to introduce the following functions. Let

$$
\left\{\begin{array}{l}
A:=\varepsilon_{1}\left(U^{\prime \prime \prime}+\left(\frac{U^{\prime}}{U}\right) U^{\prime \prime}\right) \\
B:=\varepsilon_{1}\left(-z^{\prime \prime \prime}-z^{\prime \prime}\left(\frac{U^{\prime}}{U}\right)\right)+\frac{U^{\prime}}{U^{2}} \\
C:=\varepsilon_{1}\left(-y^{\prime \prime \prime}-y^{\prime \prime}\left(\frac{U^{\prime}}{U}\right)\right)+\frac{U^{\prime}}{U^{2}}
\end{array}\right.
$$


Then the Laplacian of $G$ is:

$$
\left(\begin{array}{l}
t A \\
B+\frac{t^{2}}{2} A \\
C+\frac{t^{2}}{2} A
\end{array}\right)
$$

Now let us compute the function $g:=\langle\Delta G, G\rangle$. We have

$$
\begin{aligned}
g & =-t^{2} U^{\prime} A+\left(z^{\prime}-\frac{t^{2}}{2} U^{\prime}\right)\left(B+\frac{t^{2}}{2} A\right)-\left(y^{\prime}-\frac{t^{2}}{2} U^{\prime}\right)\left(C+\frac{t^{2}}{2} A\right) \\
& =-t^{2} U^{\prime} A+\left(z^{\prime} B\right)+\frac{t^{2}}{2}\left(z^{\prime} A-U^{\prime} B\right)+\left(-y^{\prime} C\right)+\frac{t^{2}}{2}\left(U^{\prime} C-y^{\prime} A\right) \\
& =-t^{2} U^{\prime} A+\left(z^{\prime} B-y^{\prime} C\right)+\frac{t^{2}}{2} A\left(z^{\prime}-y^{\prime}\right)+\frac{t^{2}}{2} U^{\prime}(C-B)
\end{aligned}
$$

But

$$
\left\{\begin{array}{c}
z^{\prime}-y^{\prime}=U^{\prime} \\
\text { and } \\
C-B=A .
\end{array}\right.
$$

Therefore we have $g:=\langle\Delta G, G\rangle=z^{\prime} B-y^{\prime} C$. Now the condition $\Delta G=f G$ which is equivalent to $\Delta G+\varepsilon_{1}\langle\Delta G, G\rangle G=0$, becomes:

$$
\left\{\begin{array}{l}
t A+ \\
\varepsilon_{1}\left(z^{\prime} B-y^{\prime} C\right)\left(-t U^{\prime}\right)=0 \\
\left(B+\frac{t^{2}}{2} A\right)+\varepsilon_{1}\left(z^{\prime} B-y^{\prime} C\right)\left(z^{\prime}-\frac{t^{2}}{2} U^{\prime}\right)=0 \\
\left(C+\frac{t^{2}}{2} A\right)+\left\{\left(z^{\prime} B-y^{\prime} C\right)\left(y^{\prime}-\frac{t^{2}}{2} U^{\prime}\right)=0 .\right.
\end{array}\right.
$$

The direct computation of the left-hand member of each the these relations gives a polynomial in $t$ with functions of $s$ as the coefficients. Thus by adjusting the power we get the equivalent following condition

$$
\left\{\begin{array}{l}
A-\varepsilon_{1}\left(z^{\prime} B-y^{\prime} C\right) U^{\prime}=0 \\
B+\varepsilon_{1}\left(z^{\prime} B-y^{\prime} C\right) z^{\prime}=0 \\
C+\varepsilon_{1}\left(z^{\prime} B-y^{\prime} C\right) y^{\prime}
\end{array}\right.
$$


But since $A=C-B$, and $U^{\prime}=z^{\prime}-y^{\prime}$, This condition is equivalent to the following

$$
\left\{\begin{array}{l}
-\left(1+\varepsilon_{1}\left(z^{2}-y^{\prime} z^{\prime}\right)\right) B+\left(1-\varepsilon_{1}\left(y^{2}-y^{\prime} z^{\prime}\right)\right) C=0 \\
\left(1+\varepsilon_{1} z^{\prime 2}\right) B-\varepsilon_{1} y^{\prime} z^{\prime} C=0 \\
\varepsilon_{1} y^{\prime} z^{\prime} B+\left(1-\varepsilon_{1} y^{\prime 2}\right) C=0 .
\end{array}\right.
$$

By multiplying the second equation by -1 and adding it to third equation we get the first equation. And by using the relation

$$
\left(r^{\prime}\right): y^{\prime 2}-z^{\prime 2}=\varepsilon_{1},
$$

the condition above, that $M^{\prime}$ is pointwise 1-type Gauss map, is now

$$
\left\{\begin{array}{l}
y^{\prime}\left(y^{\prime} B-z^{\prime} C\right)=0 \\
z^{\prime}\left(y^{\prime} B-z^{\prime} C\right)=0 .
\end{array}\right.
$$

Now let us compute the quantity $y^{\prime} B-z^{\prime} C$. With the $B$ and $C$ given by 4.5 and $U^{\prime}=z^{\prime}-y^{\prime}$, we have.

$$
\left\{\begin{array}{l}
y^{\prime} B-z^{\prime} C \\
=y^{\prime}\left(\varepsilon_{1}\left(-z^{\prime \prime \prime}-z^{\prime \prime}\left(\frac{U^{\prime}}{U}\right)\right)+\frac{U^{\prime}}{U^{2}}\right)-z^{\prime}\left(\varepsilon_{1}\left(-y^{\prime \prime \prime}-y^{\prime \prime}\left(\frac{U^{\prime}}{U}\right)\right)+\frac{U^{\prime}}{U^{2}}\right) \\
=\varepsilon_{1}\left(\left(z^{\prime} y^{\prime \prime \prime}-y^{\prime} z^{\prime \prime \prime}\right)+\frac{U^{\prime}}{U}\left(z^{\prime} y^{\prime \prime}-y^{\prime} z^{\prime \prime}\right)\right)-\frac{U^{\prime}}{U^{2}}\left(z^{\prime}-y^{\prime}\right) \\
=\varepsilon_{1}\left(\left(z^{\prime} y^{\prime \prime \prime}-y^{\prime} z^{\prime \prime \prime}\right)+\frac{U^{\prime}}{U}\left(z^{\prime} y^{\prime \prime}-y^{\prime} z^{\prime \prime}\right)\right)-\frac{U^{\prime 2}}{U^{2}}
\end{array}\right.
$$

Now consider the term $U^{\prime}\left(z^{\prime} y^{\prime \prime}-y^{\prime} z^{\prime \prime}\right)$. By using the relation $\left(r^{\prime}\right)$ : $y^{\prime 2}-z^{\prime 2}=\varepsilon_{1}$ and its derivative one get easily that

$$
U^{\prime}\left(z^{\prime} y^{\prime \prime}-y^{\prime} z^{\prime \prime}\right)=\varepsilon_{1} U^{\prime \prime}
$$

Then $y^{\prime} B-z^{\prime} C$ becomes

$$
\left\{\begin{array}{l}
y^{\prime} B-z^{\prime} C=\varepsilon_{1}\left(\left(z^{\prime} y^{\prime \prime \prime}-y^{\prime} z^{\prime \prime \prime}\right)+\frac{U^{\prime \prime}}{U}-\frac{U^{\prime 2}}{U^{2}}\right. \\
=\varepsilon_{1}\left(\left(z^{\prime} y^{\prime \prime \prime}-y^{\prime} z^{\prime \prime \prime}\right)+\left(\frac{U^{\prime}}{U}\right)^{\prime} .\right.
\end{array}\right.
$$

Finally, by using this with the relation

$$
2 H^{\prime}=\varepsilon_{1}\left(\left(z^{\prime} y^{\prime \prime \prime}-y^{\prime} z^{\prime \prime \prime}\right)+\left(\frac{U^{\prime}}{U}\right)^{\prime}\right.
$$

we see that the condition $(\star \star)$ above is:

$$
\left\{\begin{array}{c}
y^{\prime} H^{\prime}=0 \\
z^{\prime} H^{\prime}=0 .
\end{array}\right.
$$

Now we can conclude as in Step 1 that $H$ is constant on $M^{\prime}$. The converse is obtained easily as in Step1. A this prove the theorem. 


\section{References}

[1] L. J. Alías, A. Ferrández, P. Lucas and M. A. Meroñno, On the Gauss map of B-scrolls, Tsukuba J. Math. 22 (1998), 317-377.

[2] B.-Y. Chen, submanifolds of finite type, Soochow J. Math. 9 (1987), 65-81.

[3] _ Total mean curvature and submanifolds of finite type, World Scientific, Singapore, 1984.

[4] B.-Y. Chen and P. Piccinni, Submanifolds with finite type Gauss map, Bull. Austral. Math. Soc. 35 (1987), 161-186.

[5] S. M. Choi, On the Gauss map of ruled surfaces in a three-dimensional Minkowski space, Tsukuba J. Math. 19 (1995), 285-304.

[6] M. Choi and Y. H. Kim, Characterization of the helicoid as ruled surfaces with pointwise 1-type Gauss map, Bull. Korean Math. Soc. 38 (2001), no. 4, 753-761.

[7] Jun-ichi Hano and K. Nomizu, On Isometric immersions of the hyperbolic plane into the Lorentz-Minkowski space and the Monge-Ampre equation of a certain type, Math. Ann. 262 (1983), 245-253.

[8] Y. H. Kim and D. W. Yoon, Ruled surfaces with pointwise 1-type Gauss map, J. Geom. Phys. 34 (2000), 191-205.

[9] A. Niang, Rotation surfaces with pointwise 1-type Gauss map (submitted for publication in 2003).

Universite' Montpellier II

case 051, Montpellier cedx 5

France

E-mail: niang@darboux.math.univ-montp2.fr 
